\title{
Various Constructions of Qudit SWAP Gate
}

\author{
S. Balakrishnan \\ Materials Physics Division, School of Advanced Sciences, VIT University, Vellore 632014, India \\ Correspondence should be addressed to S. Balakrishnan; physicsbalki@gmail.com
}

Received 27 May 2014; Revised 15 July 2014; Accepted 16 July 2014; Published 5 August 2014

Academic Editor: Franco Nori

Copyright (C) 2014 S. Balakrishnan. This is an open access article distributed under the Creative Commons Attribution License, which permits unrestricted use, distribution, and reproduction in any medium, provided the original work is properly cited.

It is known that SWAP operation for quantum systems of arbitrary dimension can be implemented using various qudit gates. In this paper, equivalence of some important qudit operations is demonstrated. Equivalence of the qudit operations is exploited to minimize the circuit complexity in the previously proposed qudit SWAP gate constructions. Further, constructions of qudit SWAP operation with minimum number of qudit gates are also proposed. Importantly, these circuit constructions of SWAP retain its properties like symmetry and simplicity.

\section{Introduction}

The fundamental unit of quantum information processing is qubit which can be realized by two-level quantum mechanical systems. The two-dimensional Hilbert space representing a single qubit can be extended to higher dimensions of $2^{n}$ with $n$ being the number of qubits. Nevertheless, the dimension of the Hilbert space is not necessarily a power of two and hence one may be interested in studying Hilbert space of arbitrary dimension $d$. Quantum systems of $d$ level are known as qudits which can be used for quantum information processing.

Two-qubit operations (also known as two-qubit gates) are fundamental in the realization of universal quantum circuits [1]. Some well-known two-qubit gates are Controlled NOT, shortly CNOT, and SWAP. While the former gate performs the NOT operation on the target qubit depending upon the control qubit, the latter gate interchanges the control-qubit input state and the target-qubit input state. It is important to mention that CNOT gate along with a few single qubit gates are capable of generating controlled unitary gates $[2,3]$. Notably, a sequence of three CNOT gates can implement SWAP operation. One of the key issues in quantum computing is the CNOT complexity in the gate constructions. Over the years, efforts have been made to reduce the gate count in the circuit constructions $[4,5]$

Extension of two-qubit operation to qudit operation is not always straightforward. In particular, retaining the features of two-qubit operation in the generalization to qudit operation is not easy. One such instance is the implementation of SWAP operation in the qudit setting. A few generalizations of CNOT operation to the qudit level are proposed with the aim to implement qudit SWAP operation. However, the number of gates utilized in the previously proposed circuit constructions of SWAP gate is not less than four [6-11]. It is shown that three copies of controlled qudit gates are sufficient to implement qudit SWAP [9]. More recently, optimal construction of qudit SWAP gate using generalized CNOT is shown by Wilmott and Wild [12].

SWAP operation has been employed in circuit designs of many quantum operations (e.g., [13]). Therefore, it is necessary to implement the SWAP operation in an optimal way in terms of gate count and operating time. Hence circuit constructions of SWAP gate using other quantum gates are proposed to achieve the task of optimality. It is interesting to note that SWAP gate, which does not produce any entanglement, is constructed with gates capable of producing entanglement upon acting on suitable input states.

First of all, the existence of equivalence between some of the qudit gates is shown. Then the equivalence of gates is exploited to minimize the number of gates used in the previously proposed qudit SWAP constructions. It is shown that the number of single qudit gates in the construction of qudit SWAP can be reduced. Such constructions would be useful in the case of high $d$-level system, for which a single-qudit gate is not easy to realize in experiments. Further, various new constructions of qudit SWAP operation with 
lesser number of gates are also proposed. It is important to mention that the new circuits for SWAP gate retain its symmetric property and simplicity in terms of gate count.

\section{Some Known Qudit Operations}

Before introducing some known qudit gates, it is necessary to define important two-qubit gates, namely, CNOT and SWAP. One of the qubits of CNOT gate is known as control and the other one is called target. Whenever the control qubit is in $|1\rangle$ state, the target qubit changes its value from $|0\rangle$ to $|1\rangle$ or from $|1\rangle$ to $|0\rangle$. The CNOT operation is given by

$$
\mathrm{CNOT}|x\rangle|y\rangle=|x\rangle|x \oplus y\rangle,
$$

where the symbol $\oplus$ represents the addition modulo two. The circuit symbol for CNOT is shown in Figure 1(a) where the dot represents the control and the symbol $\oplus$ represents the target. The SWAP gate interchanges the input states, say, $|\phi\rangle$ and $|\psi\rangle$. The action of SWAP is given by

$$
\text { SWAP }|\phi\rangle|\psi\rangle=|\psi\rangle|\phi\rangle \text {. }
$$

The circuit symbol for two-qubit SWAP gate is shown in Figure 1(b). Note that the input states can also be $d$ dimensional and hence we have qudit SWAP operation.

Now we introduce some qudit gates which are the different versions of qudit CNOT operation. Among the various versions, $C X_{d}$ is widely used and the operations of $C X_{d}$ are given by

$$
C X_{d}|x\rangle|y\rangle=|x\rangle|x+y\rangle
$$

with modulo $d$ addition. Note that throughout the paper we consider addition and subtraction of modulo $d$. The inverse of the above operation is

$$
C X_{d}^{\dagger}|x\rangle|y\rangle=|x\rangle|y-x\rangle
$$

with modulo $d$ subtraction. It is worth noting that $C X_{d} \neq$ $C X_{d}^{\dagger}$, implying that the gates are not Hermitian. This is the main issue in the qudit generalization of CNOT. With the aim of having Hermitian qudit CNOT operation, GXOR is introduced. The action of GXOR is as follows:

$$
G X O R|x\rangle|y\rangle=|x\rangle|x-y\rangle .
$$

Recently, $C \widetilde{X}$ gate is introduced with the aim of proposing a simpler qudit SWAP gate [11]. The action of $C \widetilde{X}$ gate is given as follows:

$$
C \widetilde{X}|x\rangle|y\rangle=|x\rangle|-x-y\rangle .
$$

Apart from these gates, we have

$$
X_{d}|x\rangle=|d-x\rangle=|-x\rangle .
$$

This gate provides modulo $d$ complement of the input state.

\section{Equivalence of Various Qudit Gates}

Equivalence of the various qudit gates is identified and mentioned in this section. The equivalence of the operation can be checked by the action of the gates on the input states $|\phi\rangle$ and $|\psi\rangle$ of arbitrary dimension $d$ :

$$
\begin{gathered}
G X O R_{1,2} \equiv X_{d 2} C X_{d 1,2}^{\dagger}, \\
G X O R_{1,2} \equiv C X_{d 1,2} X_{d 2}, \\
G X O R_{2,1} \equiv X_{d 1} C X_{d 2,1}^{\dagger}, \\
G X O R_{2,1} \equiv C X_{d 2,1} X_{d 1}, \\
C \widetilde{X}_{1,2} \equiv X_{d 2} C X_{d 1,2}, \\
C \widetilde{X}_{1,2} \equiv C X_{d 1,2}^{\dagger} X_{d 2}, \\
C \widetilde{X}_{2,1} \equiv X_{d 1} C X_{d 2,1}, \\
C \widetilde{X}_{2,1} \equiv C X_{d 2,1}^{\dagger} X_{d 1} .
\end{gathered}
$$

Using the equivalence given by (8) and (13), it can be shown that

$$
\mathrm{GXOR}_{1,2} \equiv X_{d 2} C \widetilde{X}_{1,2} X_{d 2} .
$$

In the similar fashion, it can be shown using the equivalence given by (11) and (14) that

$$
\mathrm{GXOR}_{2,1} \equiv X_{d 1} C \widetilde{X}_{2,1} X_{d 1} .
$$

\section{Qudit SWAP Operation}

It is known that the concatenation of three CNOT gates, can implement two-qubit SWAP operation. The CNOT swapping circuit is shown in Figure 2. Note that the control and target qubits are interchanged in the second gate.

Before analyzing the qudit SWAP circuits, consider a special case. That is, we have SWAP circuits if one of the initial states is known. The circuit in Figure 3 can swap the state $|\phi\rangle|0\rangle$ into $|0\rangle|\phi\rangle[6]$.

Other possibilities of swapping the state $|\phi\rangle|0\rangle$ using GXOR, $C X_{d}$, and $C X_{d}^{\dagger}$ gates are shown in Figure 4. Note that all the circuits shown in Figure 4 consist of only two basic qudit gates.

The equivalence of qudit gates identified in the last section can be exploited to simplify the existing circuits for the qudit SWAP gate.

The qudit SWAP gate circuit shown in Figure 5 is proposed in $[7,10]$. Using the equivalence given by (9) and (11), we can simplify the circuit as shown in Figure 6. Note that six gates are used in the previously proposed circuit (see Figure 5), whereas only four gates were employed in the new circuit (Figure 6). Similarly, qudit SWAP can be realized with $C X_{d}, C X_{d}^{\dagger}$, and $X_{d}$ gates using the circuit shown in Figure 7. This circuit can be simplified by noting down the equivalence given by (10) and the simplified circuit is shown in Figure 8 .

The qudit SWAP can be implemented with a circuit consisting of three GXOR gates and two $X_{d}$ gates $[14,15]$ and 


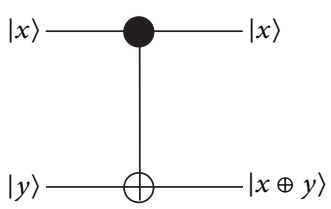

(a)

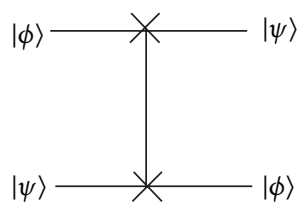

(b)

FIgUre 1: Two-qubit gates. (a) CNOT; (b) SWAP.

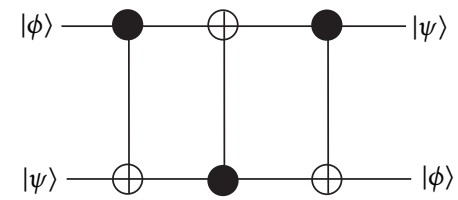

Figure 2: Implementation of qubit SWAP using CNOT.

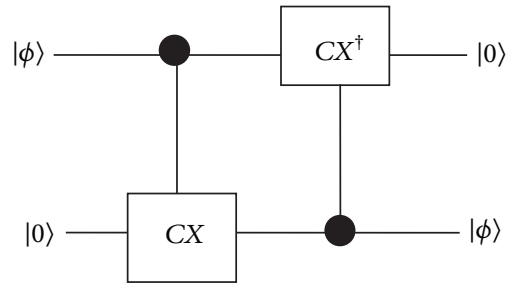

FIgURE 3: Partial qudit SWAP circuit for one known input state. Note that a general dimension $d$ is assumed and for simplicity here we have written $C X$ and $C X^{\dagger}$ as well in the subsequent figures.

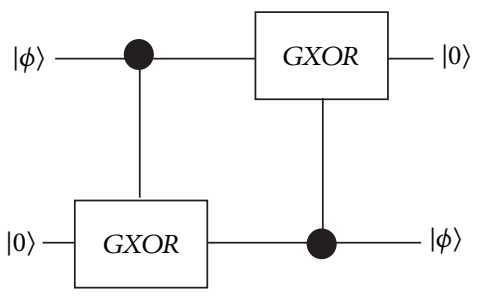

$|\phi\rangle$

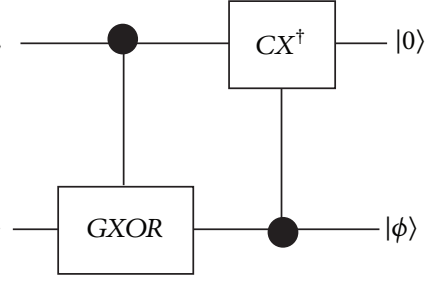

$|\phi\rangle$

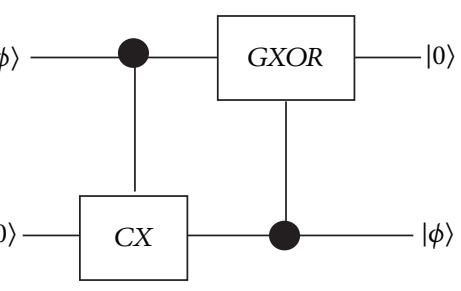

FIGURE 4: Partial qudit SWAP circuit for one known input state using $G X O R, C X_{d}$, and $C X_{d}^{\dagger}$.

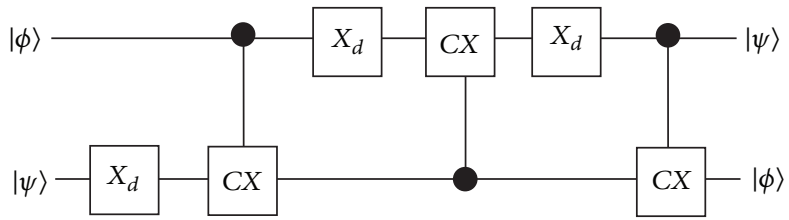

FIgURE 5: Qudit SWAP circuit using $C X_{d}$ and $X_{d}$ gates.

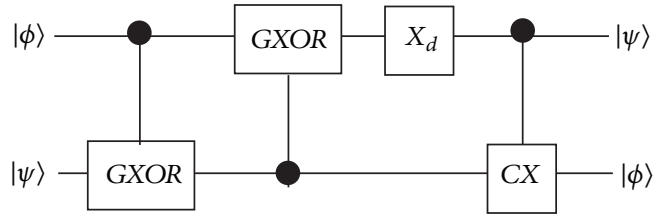

FIgURE 6: Simplified version of the circuit in Figure 5. 


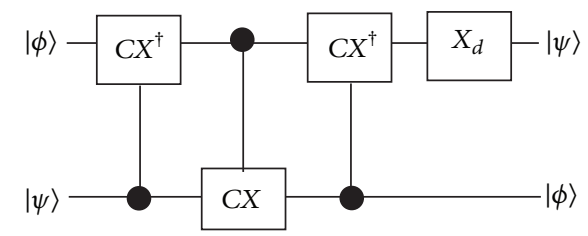

FIgURE 7: Qudit SWAP circuit using $C X_{d}, C X_{d}^{\dagger}$, and $X_{d}$ gates.

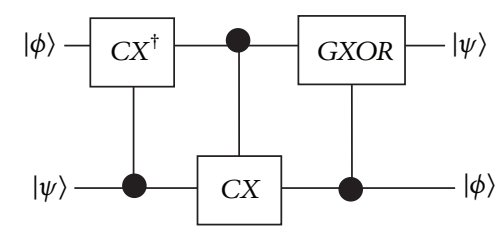

FIGURE 8: Simplified version of the circuit in Figure 7.

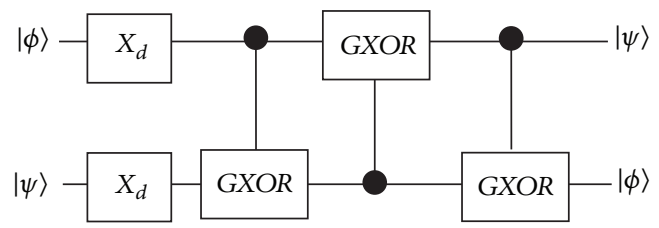

FIgURE 9: Qudit SWAP circuit with $G X O R_{d}$ and $X_{d}$ gates.

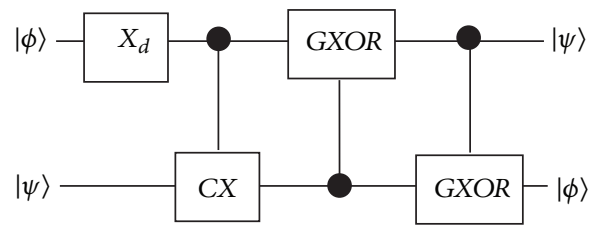

FIGURE 10: Simplified version of the circuit in Figure 9.
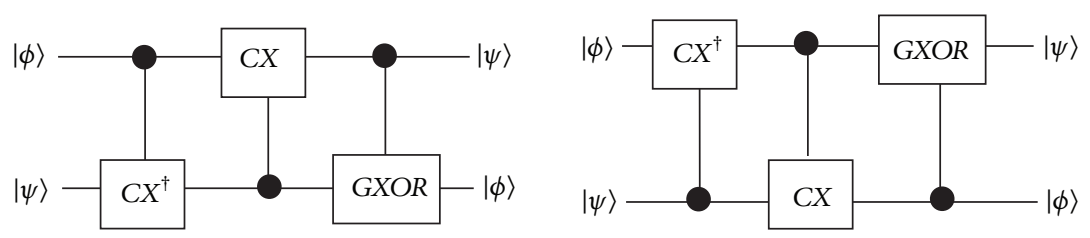

FIGURE 11: Implementation of qudit SWAP using $G X O R, C X_{d}$, and $C X_{d}^{\dagger}$ gates.
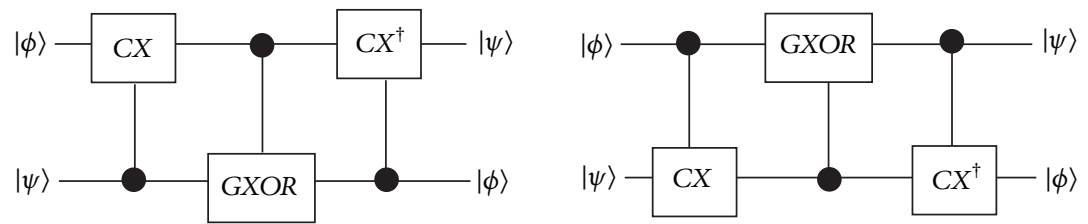

FIGURE 12: Implementation of qudit SWAP using GXOR, $C X_{d}$, and $C X_{d}^{\dagger}$ gates.
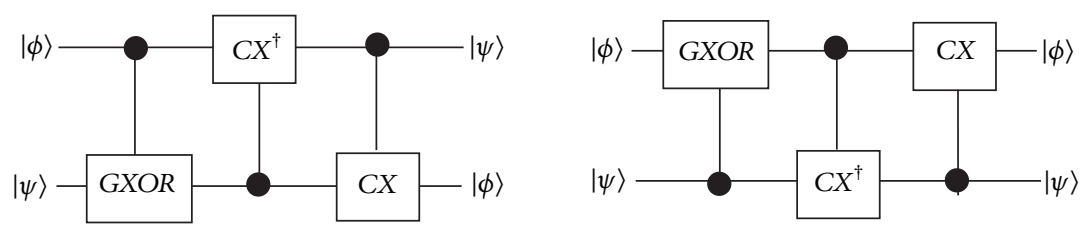

FIGURE 13: Implementation of qudit SWAP using GXOR, $C X_{d}$, and $C X_{d}^{\dagger}$ gates. 
the circuit is shown in Figure 9. Observing the equivalence given by (9) in the circuit (see Figure 9), it is possible to simplify the circuit as shown in Figure 10.

Apart from simplifying the previously proposed circuits, the equivalence between the qudit gates can be employed to introduce more qudit SWAP circuits. The qudit SWAP can be constructed with three basic gates $G X O R, C X_{d}$, and $C X_{d}^{\dagger}$. The equivalence of SWAP operation is given by (18a)(18b) and corresponding circuit is shown in Figure 11. As the circuit can implement SWAP for arbitrary input states, the inverted circuit can also implement the same operation. By cyclic rearrangement of $G X O R, C X_{d}$, and $C X_{d}^{\dagger}$ gates, more circuit constructions are possible. Such circuits are shown in Figures 12 and 13 and corresponding operation equivalence is given by (19a)-(19b) and (20a)-(20b), respectively. Note that circuit and its inverted version are given in Figures 11 to 13. Consider the following:

$$
\begin{aligned}
& G X O R_{1,2} C X_{d 2,1} C X_{d 1,2}^{\dagger} \equiv \text { SWAP, } \\
& G X O R_{2,1} C X_{d 1,2} C X_{d 2,1}^{\dagger} \equiv \text { SWAP, } \\
& C X_{d 2,1}^{\dagger} G X O R_{1,2} C X_{d 2,1} \equiv \text { SWAP, } \\
& C X_{d 1,2}^{\dagger} G X O R_{2,1} C X_{d 1,2} \equiv \text { SWAP, } \\
& C X_{d 1,2} C X_{d 2,1}^{\dagger} G X O R_{1,2} \equiv \text { SWAP, } \\
& C X_{d 2,1} C X_{d 1,2}^{\dagger} G X O R_{2,1} \equiv \text { SWAP. }
\end{aligned}
$$

Therefore the qudit GXOR gate can be combined with $C X_{d}$ and $C X_{d}^{\dagger}$ in a cyclic fashion to produce qudit SWAP operation. It is known from previous results $[7,10,14,15]$ that qudit SWAP operation is constructed with not less than four gates. In this work, the possibility of constructing qudit SWAP operation with three qudit gates is indicated. However, three copies of $C \widetilde{X}$ gate are shown to be sufficient to achieve qudit SWAP operation [11]. Note that $C \widetilde{X}$ gate can be decomposed into two Quantum Fourier Transforms and a phase gate, which can be realized for multilevel atoms $[11,16]$.

\section{Conclusion}

In this work, the existence of equivalence between various qudit gates is illustrated and these gates are useful in the construction of qudit SWAP circuit. The identification of equivalence of the qudit operations is useful to minimize the circuit complexity in the previously proposed qudit SWAP gate constructions. In particular, the number of single qudit gates is minimized in the qudit SWAP gate construction. These constructions are useful whenever the single qudit gates are difficult to implement in the experiment. Moreover, many new qudit SWAP circuits comprising of lesser number of gates are proposed. Notably, the proposed circuits have the property of symmetry thereby the inverted circuits also implement qudit SWAP operation. Hence, we argue that not only the three copies of controlled qudit gate $\left(C \widetilde{X}_{d}\right)$ can implement SWAP but also combinations of three different versions of controlled qudit gates ( $G X O R, C X_{d}$, and $C X_{d}^{\dagger}$ ) can do the same. In short, we have presented various qudit circuits which can be employed in the construction of complex qudit circuits.

\section{Conflict of Interests}

The author declares that there is no conflict of interests regarding the publication of this paper.

\section{References}

[1] A. Barenco, C. H. Bennett, R. Cleve et al., "Elementary gates for quantum computation," Physical Review A, vol. 52, no. 5, pp. 3457-3467, 1995.

[2] M. A. Nielsen and I. L. Chuang, Quantum Computation and Quantum Information, Cambridge University Press, Cambridge, UK, 2003.

[3] A. Galindo and M. A. Martin-Delgado, "Information and computation: classical and quantum aspects," Reviews of Modern Physics, vol. 74, no. 2, pp. 347-423, 2002.

[4] V. V. Shende, I. L. Markov, and S. S. Bullock, "Minimal universal two-qubit controlled-NOT-based circuits," Physical Review A, vol. 69, no. 6, 2004.

[5] G. Vidal and C. M. Dawson, "Universal quantum circuit for two-qubit transformations with three controlled-NOT gates," Physical Review A, vol. 69, Article ID 010301, 2004.

[6] N. D. Mermin, "From classical state swapping to quantum teleportation," Physical Review A, vol. 65, no. 1, Article ID 012320, 2001.

[7] K. Fujii, "Exchange gate on the qudit space and Fock space," Journal of Optics B, vol. 5, no. 6, pp. S613-S618, 2003.

[8] C. M. Wilmott, "On swapping the states of two qudits," International Journal of Quantum Information, vol. 9, no. 6, pp. 15111517, 2011.

[9] C. Wilmott and P. Wild, "On a generalized quantum swap gate," International Journal of Quantum Information, vol. 10, no. 3, Article ID 1250034, 18 pages, 2012.

[10] G. Paz-Silva, S. Rebić, J. Twamley, and T. Duty, "Perfect mirror transport protocol with higher dimensional quantum chains," Physical Review Letters, vol. 102, Article ID 020503, 2009.

[11] J. C. Garcia-Escartin and P. Chamorro-Posada, "A SWAP gate for qudits," Quantum Information Processing, vol. 12, no. 12, pp. 3625-3631, 2013.

[12] C. Wilmott and P. R. Wild, "Towards an optimal swap gate," Quantum Information Processing, vol. 13, no. 6, pp. 1467-1482, 2014.

[13] L. Liang and C. Li, "Realization of quantum SWAP gate between flying and stationary qubits," Physical Review A, vol. 72, Article ID 024303, 2005.

[14] X. Wang, "Continuous-variable and hybrid quantum gates," Journal of Physics A: Mathematical and General, vol. 34, no. 44, pp. 9577-9584, 2001.

[15] G. Alber, A. Delgado, N. Gisin, and I. Jex, "Efficient bipartite quantum state purification in arbitrary dimensional Hilbert spaces," Journal of Physics A, vol. 34, no. 42, pp. 8821-8833, 2001.

[16] A. Muthukrishnan and C. R. Stroud Jr., "Quantum fast Fourier transform using multilevel atoms," Journal of Modern Optics, vol. 49, no. 13, pp. 2115-2127, 2002. 

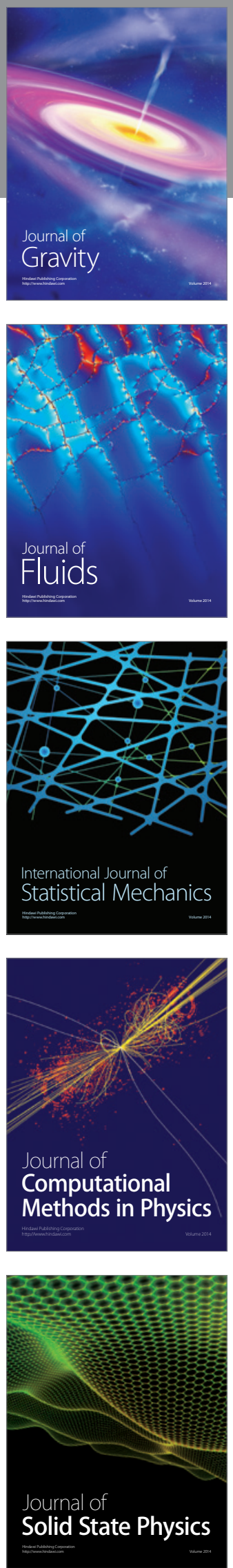

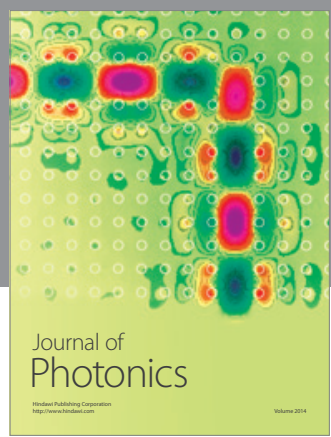

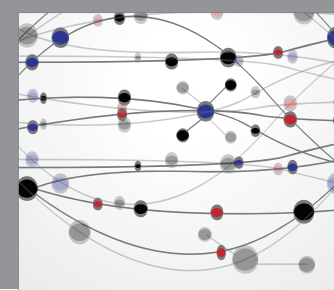

The Scientific World Journal

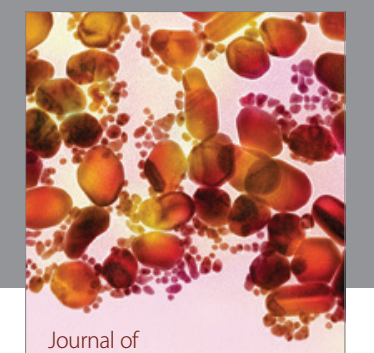

Soft Matter
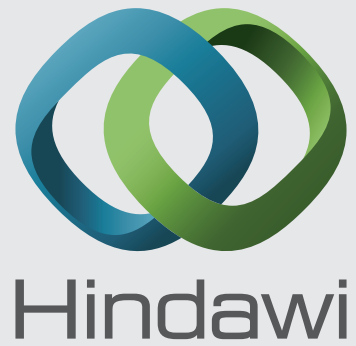

Submit your manuscripts at

http://www.hindawi.com
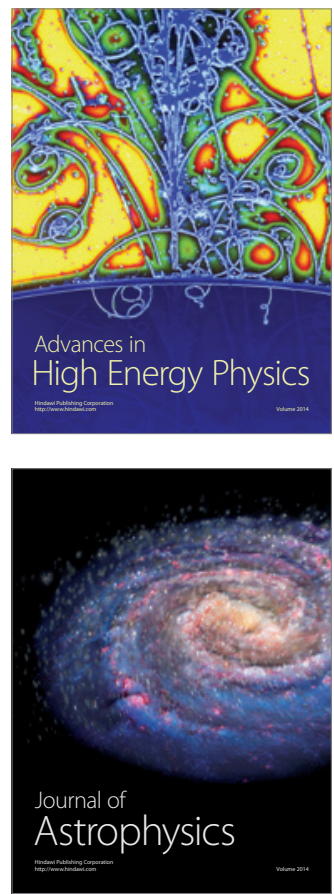
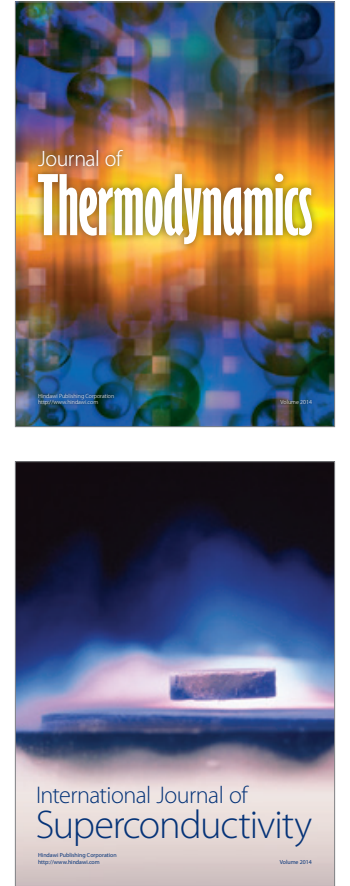
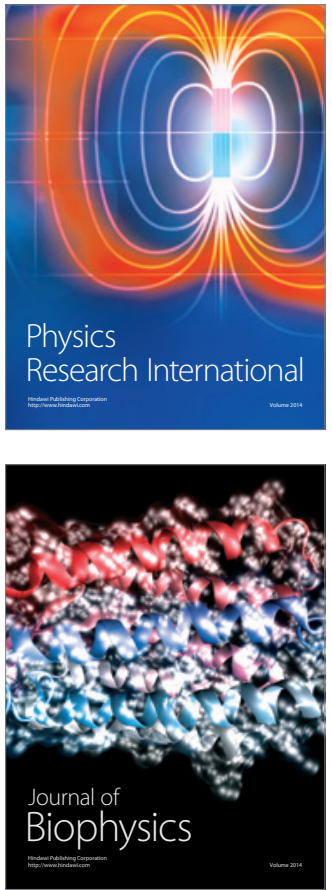
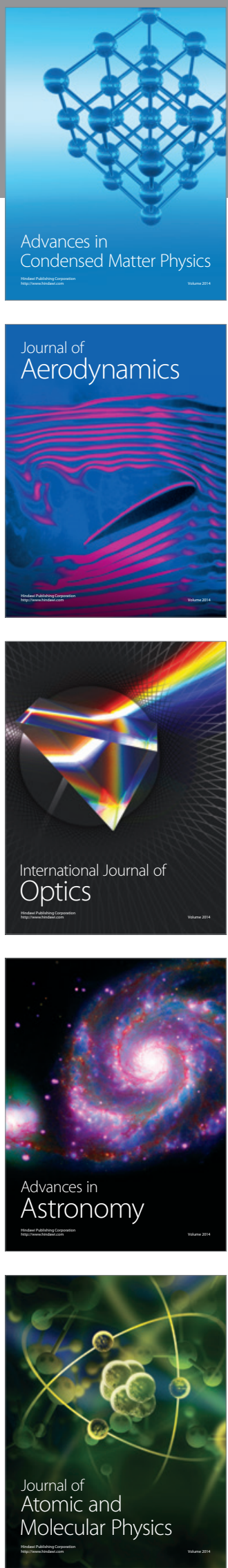